Journal of Clinical Investigation

Vol. 42, No. 10, 1963

\title{
MECHANICAL AND CHEMICAL PROPERTIES OF ARTERIES IN EXPERIMENTAL HYPERTENSION *
}

\author{
By ERIC O. FEIGL, $\dagger$ LYSLE H. PETERSON, AND ALLAN W. JONES
}

(From the Department of Physiology, University of Pennsylvania School of Medicine, Philadelphia, $P a$.)

(Submitted for publication March 13, 1963; accepted July 3, 1963)

There is general agreement that the final common path in the etiology of arterial hypertension is the arterial wall. A net decrease in the radius of the arterial tree is primarily responsible for the increased peripheral resistance characteristic of hypertension. The radius that an artery assumes is, of course, due to the combined effects of the distending pressure and the mechanical stiffness ("tone") of the wall.

Since vascular smooth muscle is responsible for physiological or normal changes in vascular stiffness or tone, increased contraction of vascular muscle has been assumed to initiate the increased tone in hypertension. Recently, it has been shown that arterial hypertension is associated with an increase in the concentration of certain ions and of the water content of the arterial wall (1).

Techniques have now been developed whereby the mechanical properties of arteries can be quantitatively evaluated in the intact living animal. Thus, it is posible to evaluate the changes in arterial tone in such animals with hypertension. This report describes a study designed to compare the mechanical properties in vivo of femoral arteries and the water and electrolyte content of these arteries before and after the development of experimental hypertension in dogs.

\section{METHODS}

Experimental design. Stock mongrel dogs were selected for the study on the basis of adequate size (over $12 \mathrm{~kg}$ ), apparent good health, and middle age. Early in the study, pressures and vessel-wall properties were obtained during general anesthesia. ${ }^{1}$ Later, dogs were trained so that pressures were obtainable on unanesthe-

* Supported in part by U. S. Public Health Service grant H-3481 (C-4) and Office of Naval Research contract N ONR 551 (18).

$\dagger$ Investigation performed during tenure of Postdoctoral Fellowship HF 10627, National Heart Institute.

1 Dial, or 5.5-diallylbarbituric acid; mixture of pentobarbital sodium with morphine before anesthesia. tized, tranquil animals, and vessel-wall properties were determined with only local anesthesia ${ }^{2}$ of the skin and subcutaneous tissues. No anesthetic was introduced into or around the vessel wall. Control measurements of pressure, vessel-wall strain, and chemical composition were made on one femoral artery. The femoral segment remained interrupted after the study, but adequate circulation in the limb persisted through collateral channels.

After the establishment of normal control values of blood pressure and vessel mechanical and chemical properties, both kidneys were wrapped with cellophane, and arterial hypertension resulted (2). After an elevation of mean blood pressure of at least $30 \mathrm{~mm} \mathrm{Hg}$ for at least 4 weeks, the measurements of vessel-wall mechanical properties and of water and electrolyte content were repeated on the contralateral vessel. Thus, each dog served as its own control, with measurements made on the femoral artery before and during hypertension.

Analysis of arterial-zoall mechanical properties and intra-arterial pressure. Intra-arterial pressure was determined directly by coupling the arterial lumen to a Technitrol capacitance manometer through a 27-gauge needle attached to the manometer with a short length of flexible plastic tubing. When only intra-arterial pressure was desired, the needle was introduced into the femoral artery percutaneously. Mean pressures were obtained by playing the output from the tape-recorded pressure curves into an averaging circuit of an analog computer. The time constant of the network was 2 seconds.

When both vessel-wall properties and pressure were required, a length of femoral artery was exposed to permit the attachment of transducers for simultaneous measurement of intra-arterial pressure and vessel diameter. The outputs of these transducers of arterial stress and strain were recorded on magnetic tape and were later replayed and recorded on an oscillograph for analysis and computation of the vessel elastic modulus. The over-all characteristics of the pressure measurements were: 1) linear, from 0 to $300 \mathrm{~mm} \mathrm{Hg}, 2)$ precision, $\pm 1 \mathrm{~mm} \mathrm{Hg}$, and 3) frequency response flat, from 0 to 30 cycles per second. Details of computing vessel-wall strain and vessel moduli have been described previously. Computation of vesselwall moduli is estimated to be reliable at better than $\pm 2 \%$ (3).

The modulus $E_{p}$ represents the "distensibility" of the arterial segment, or the relationship between the intraarterial pressure and the diameter of the artery. This

\footnotetext{
2 Procaine hydrochloride.
} 
TABLE I

Femoral artery determinations in normotensive dogs*

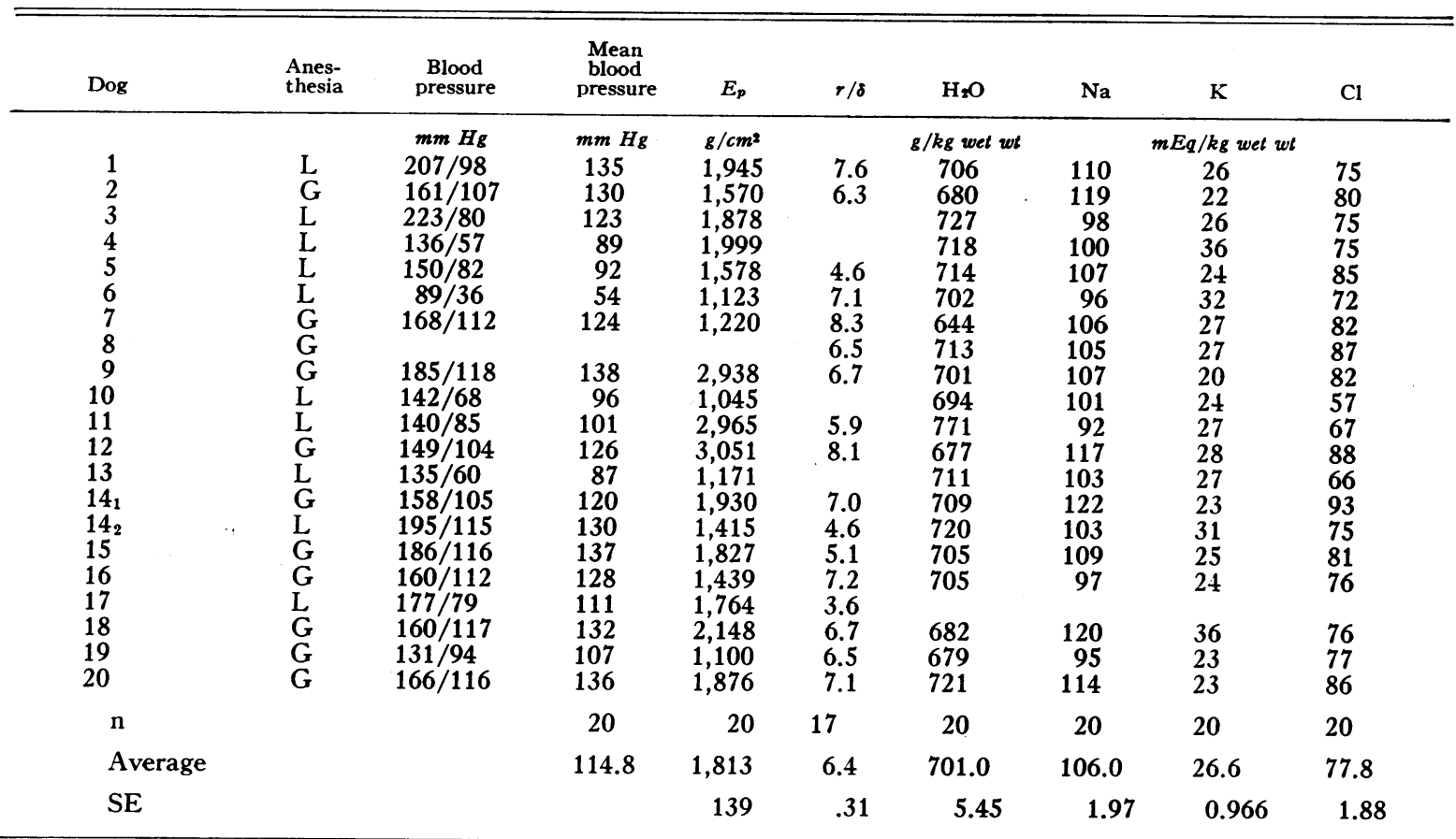

${ }^{*} \mathrm{~L}=$ local anesthesia (procaine), $\mathrm{G}=$ general anesthesia (Dial), $E_{p}=$ relationship between pressure and vessel strain; and $r / \delta=$ vessel radius/wall thickness; $n=$ number in series.

TABLE II

Femoral artery determinations in hypertensive dogs*

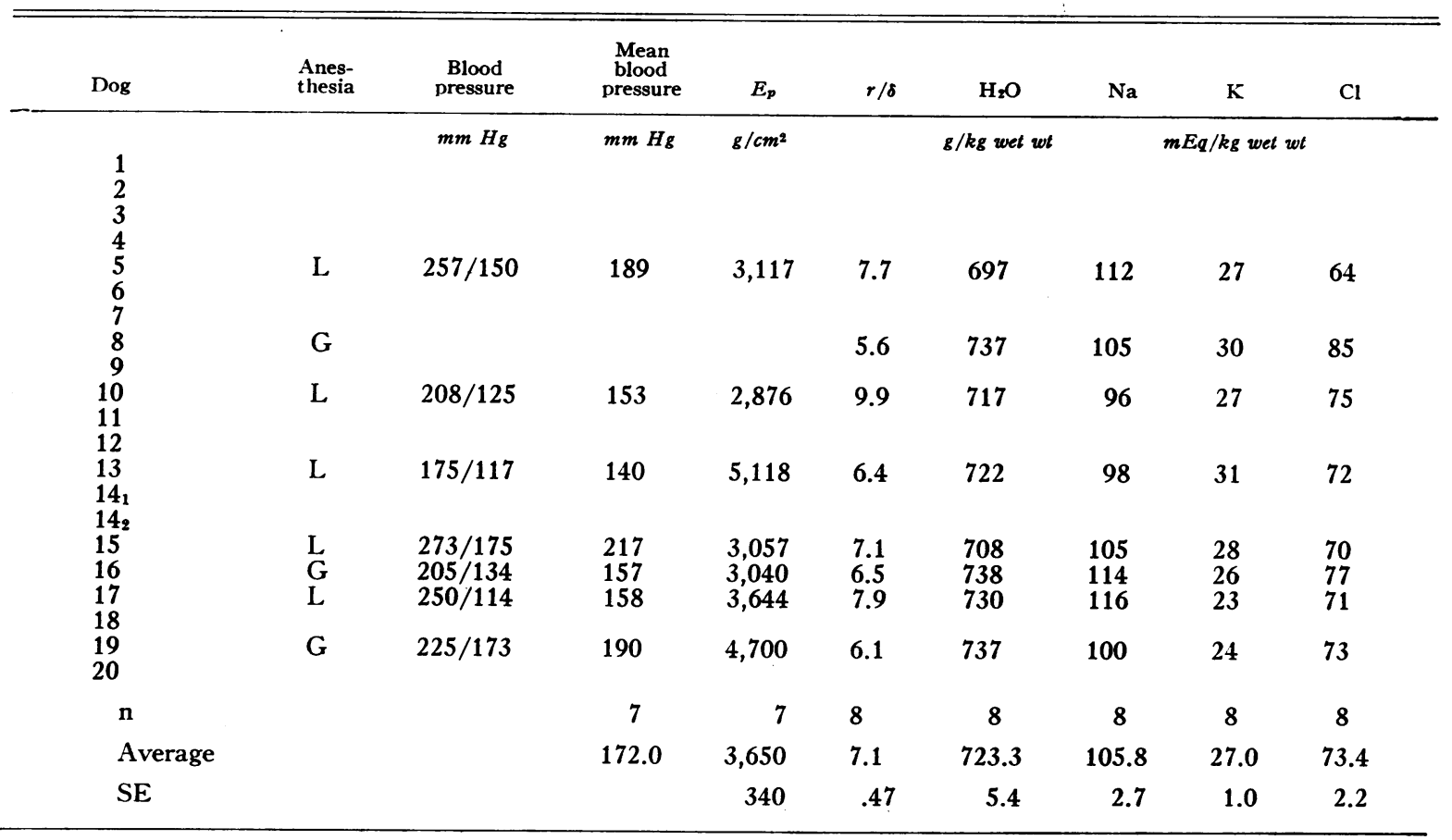

* Symbols as in Table I. 
modulus is different from the modulus of stiffness of the vessel-wall material itself, since pressure diameter relations are due not only to wall stiffness, but also to the geometry of the vessel. Briefly, the relationship between diameter or radius and strain is : $\epsilon=\left(r-r_{0}\right) / r_{0}$, where $\epsilon$ is strain, $r$ is the instantaneous radius, and $r_{0}$ is the radius at zero stress. The relationship between pressure and strain may be expressed by $P=E_{p} \epsilon+R_{p}(d \epsilon / d t)$, where $E_{p}$ represents the relationship between pressure and vessel strain, and $R_{p}$ represents the relationship between pressure and the strain velocity.

If the stiffness of the wall material is to be evaluated, then it is necessary to determine the relationship between the wall tangential stress and wall strain. For a thinwalled vessel, the relationship between pressure, $P$, and wall tension, $T$, is $T=P r / \delta$, where $\delta$ is vessel-wall thickness. Therefore, the elastic and viscous moduli, $E$ and $R$, respectively, are given by, $P(r / \delta)=E \epsilon+R(d \epsilon /$ $d t)$.

In Table I, the $E_{p}$ values of the control dogs are given. The modulus $E_{p}$ has the same units as pressure and is expressed in grams per square centimeter. In this study, $E_{p}$ was estimated in the form $E_{p}=$ $\Delta P / \Delta \epsilon$, where $\Delta P$ is the pulse pressure (between the end of diastole and the height of systole) and $\Delta \epsilon$ is the change in strain (per unit change in diameter) during the same period. The change in strain was taken as the change in diameter divided by the mean diameter. These computations have been discussed in detail previously (3).

It is desirable to evaluate the radius: wall-thickness ratio $(r / \delta)$, since changes in the stiffness of the vessel as a whole $\left(E_{p}\right)$ may result from either changes in the geometry of the vessel $(r / \delta)$ or changes in the wall itself, or both. Therefore, segments of the vessels were removed and reconstituted to their original length and cross-section by stretching with sutures and inflation with a balloon and then fixation in formalin-saline. The radius and wall thickness were measured from cut sections with a calibrated microscope. This method is

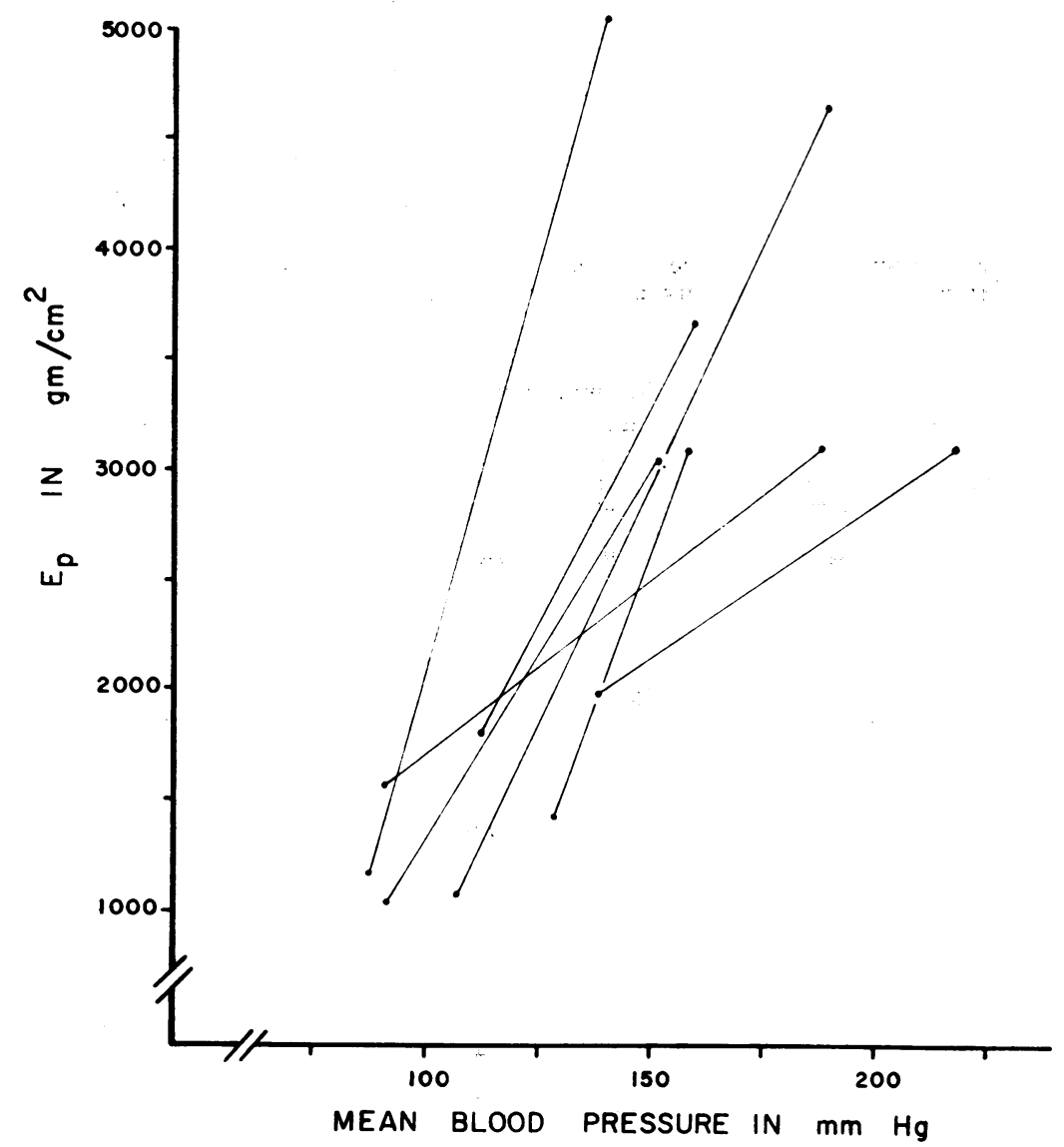

Fig. 1. A plot of mean blood pressure against elastic stiffness (E BEFORE AND DURING HYPERTENSION. Each set of two points connected by a line represents an individual dog's control and hypertensive determinations. The figure shows the consistent elevation in $E_{p}$ observed with hypertension in this study. 


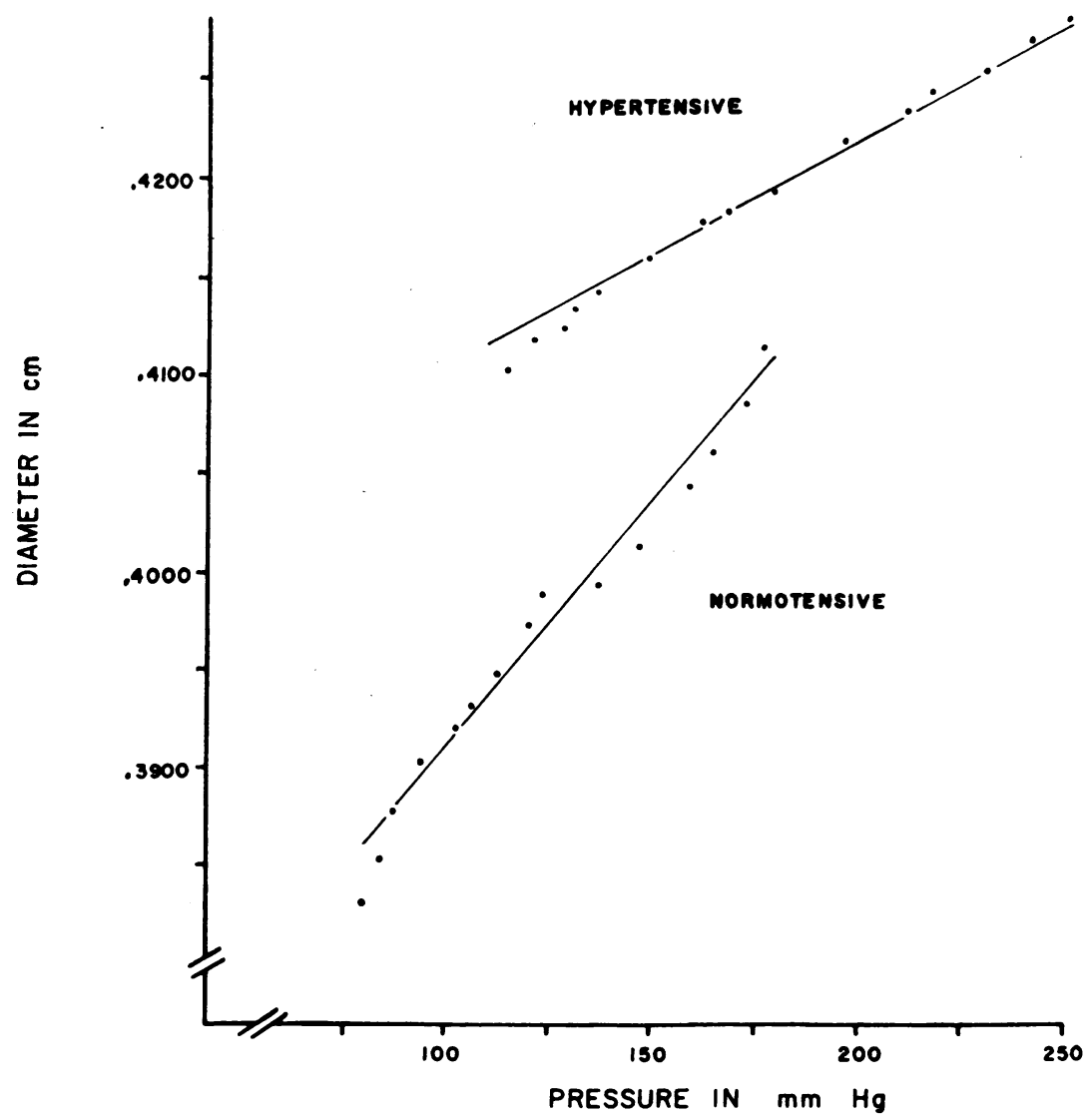

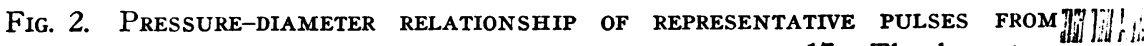
NORMOTENSIVE AND HYPERTENSIVE FEMORAL ARTERIES OF DOG 17. The hypertensive pulse was significantly nonlinear $(p<0.05)$. The normotensive pulse did not deviate significantly from linearity. The figure is presented as part of the discussion to show that increase in $E_{p}$ was not due to elastic nonlinearity (see text).

not very precise because fixing agents tend to dehydrate the tissue, but large changes in the $r / \delta$ ratio should have been detectable.

Analysis of chemical properties. A small segment of artery was excised immediately after the stress-strain measurement had been completed. The excised sample of vessel was immediately opened, the blood removed, and the vessel trimmed of attached fibrous tissue. The specimen was placed in a stoppered, tarred tube, promptly weighed, then dried at $96^{\circ} \mathrm{C}$ for 24 hours, and reweighed to yield the water content. The fat was extracted with ether; the sample was dried and reweighed for measurement of the ether-extractable fat. The specimen was then crushed to a coarse powder and reweighed. This sample was extracted with a portion of $0.75 \mathrm{~N}$ nitric acid for 72 hours. Samples of the supernatant fluid were used for $\mathrm{Na}, \mathrm{K}$, and $\mathrm{Cl}$ determinations. $\mathrm{Na}$ and $\mathrm{K}$ were measured by a flame photometer with an internal standard in the usual manner. Chloride determinations were done with a Cotlove chloride titrator by a standard technique.

\section{RESULTS}

Results in hypertensive dogs are summarized in Table II (controls are presented in Table I). Of the 20 original dogs, 8 hypertensive ones survived to a terminal study. One dog, $\mathrm{Mu}$, listed twice in Table I, survived 4 months after kidneywrapping without developing hypertension and was sacrificed for a second control determination. The type of anesthesia (general or local) used is listed. The systolic, diastolic, and mean blood pressures noted were those obtained when the wall properties were measured.

The average of 20 determinations of $E_{p}$ in 19 normal dogs was $1,813 \mathrm{~g}$ per $\mathrm{cm}^{2}$. The average of 7 determinations of $E_{p}$ in 7 hypertensive dogs was $3,650 \mathrm{~g} \mathrm{per} \mathrm{cm}^{2}$. If $E_{p}$ measurements for each of the 7 hypertensive dogs are compared with their 
own control values by a paired $t$ test, the increase is statistically significant $(p<0.005)$. If the 20 control observations are compared with the 7 hypertensive measurements, and these are considered as independent, an unpaired $t$ test gives $p<0.001$. The differences seen in $E_{p}$ in the 7 dogs with measurements before and during hypertension are plotted in Figure 1, which shows the consistent increase in elastic stiffness with hypertension. The radius: wall-thickness ratio $r / \delta$ in 17 normotensive dogs was 6.4 . In the 8 hypertensive dogs, it was 7.1. This is not a significant difference.

A test on data obtained from 6 dogs before and after they developed hypertension evaluated the stress-strain characteristics over normotensive and hypertensive ranges of strain. Magnetic tape records of blood pressure and vessel diameter were replayed and rerecorded on paper at large amplification to increase the sensitivity of measurement. Also, the paper speed was such that time could be measured accurately to 0.01 second. Fifteen synchronous pressure and diameter measurements were then made during the falling part of the pulses. In this period the effects of viscosity and hysteresis in the wall were small, and the linearity of a pressure-diameter elastic plot could be evaluated. This evaluation was made with a statistical test of linearity described by Bartlett (4). This test of linearity does not establish the principle of superposition for the vessel, or validate the linear elastic equation used, nor is it intended to. Rather, it was used to evaluate the likelihood that the changes observed before and during hypertension were due to nonlinear behavior of the vessel. In 11 of the 12 cases, the deviations from linearity were not significant. In one case, the hypertensive determina-

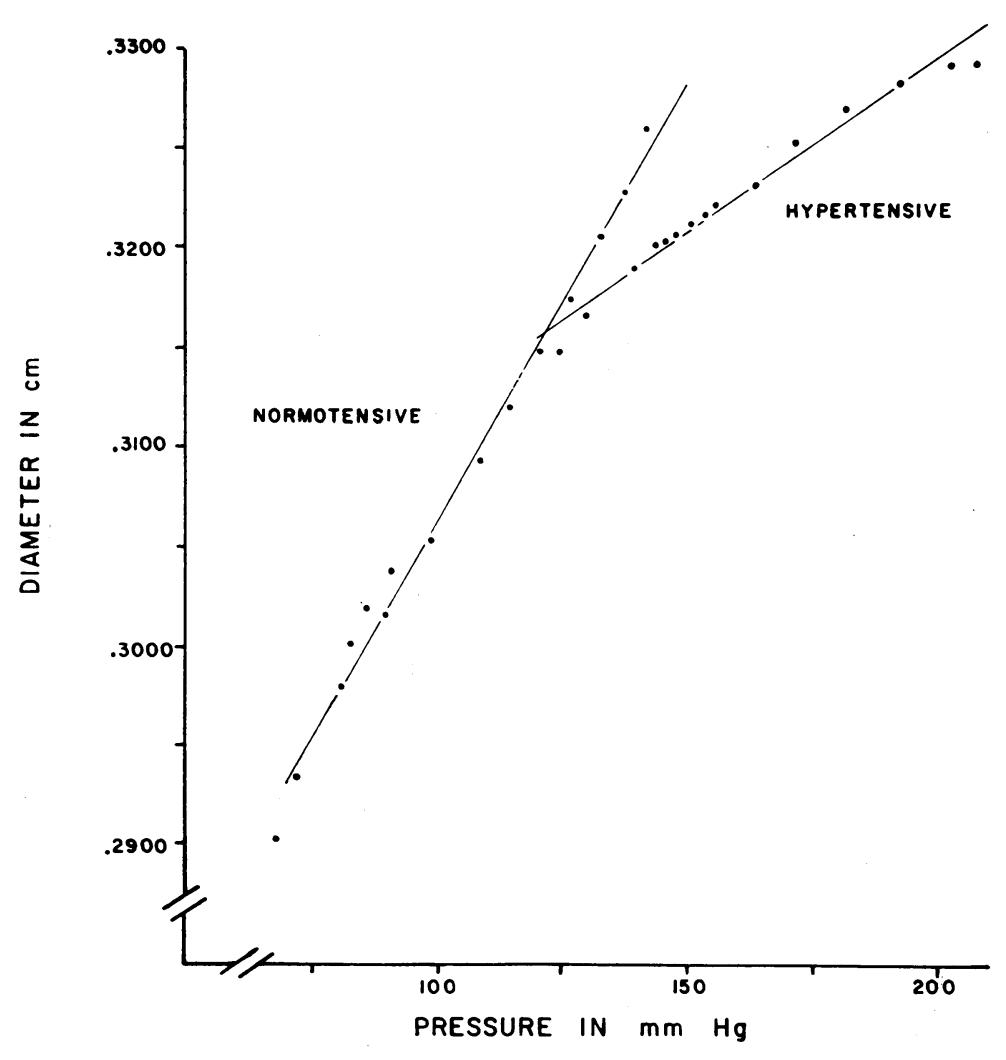

Fig. 3. Pressure-diameter Relationship of Representative PUlses FROM NORMOTENSIVE AND HYPERTENSIVE FEMORAL ARTERIES OF DOG 10. Neither line deviated significantly from linearity. The figure is presented as part of the discussion to show that increase in $E_{p}$ was not due to elastic nonlinearity (see text). 
tion of $\operatorname{dog} 17$, the deviations from linearity were significant $(p<0.05)$. This least favorable case is plotted in Figure 2. A representative case from $\operatorname{dog} 10$ is plotted in Figure 3. These figures show the pressure-diameter relationship for two typical heart beats, one normotensive and the other hypertensive.

The average of 20 determinations of the water content of normal femoral arteries was $701.0 \mathrm{~g}$ per $\mathrm{kg}$ fresh wet wt. The average of 8 determinations of the water content of hypertensive femoral arteries was $723.3 \mathrm{~g}$ per $\mathrm{kg}$ wet $\mathrm{wt}$, indicating a significant increase in the water content of femoral arteries in the hypertensive vessels $(p<0.01)$. The average sodium concentration for the normotensive group was $106.0 \mathrm{mEq}$ per $\mathrm{kg}$ wet $\mathrm{wt}$, and for the hypertensive group, $105.8 \mathrm{mEq}(\mathrm{p}=\mathrm{NS})$. The average normotensive potassium was 26.6 $\mathrm{mEq}$, and for the hypertensives, $27.0 \mathrm{mEq}$ per $\mathrm{kg}$ wet wt. $(p=\mathrm{NS})$. The average of 20 determinations on normotensive femoral arteries for chloride concentration was $77.8 \mathrm{mEq}$ per $\mathrm{kg}$ wet wt. The average of 8 determinations on hypertensive femoral arteries was $73.4 \mathrm{mEq}$ per $\mathrm{kg}$ wet wt $(\mathrm{p}=\mathrm{NS})$.

\section{DISCUSSION}

These findings relate to the changes observed in the femoral artery with the development of hypertension caused by wrapping the kidneys in cellophane, and apparently constitute the first quantitative measurements of both artery-wall stiffness and distensibility in hypertension in vivo. Mechanical properties were measured in only one segment of the vascular tree, the femoral artery. This site was chosen because it is bilaterally represented, and previous studies have shown that the two femoral arteries in the same dog normally do not differ. This site is also accessible for measurement of both mechanical and chemical properties without general anesthesia.

In all cases in this study, $E_{p}$ of the femoral artery increased with the development of hypertension. This increase in vessel stiffness will, of course, result in a smaller vessel radius for a given distending pressure. Although the largest proportion of "peripheral resistance" is distributed distal to the femoral artery, the changes observed can be assumed to be characteristic and repre- sentative of changes occurring in arteries generally.

Under normal physiological circumstances, regulation of the circulation, i.e., changes in resistance and distribution of blood flow, is achieved by variations in vascular radii. These variations are, in turn, controlled through alterations in the mechanical properties of the vessel wall that are referred to as vessel stiffness, or tone. Wall stiffness is normally altered by changes in the properties of vascular smooth muscle. These same mechanisms may become abnormally altered in disease states such as hypertension, but there are other factors that can also influence vessel-wall stiffness.

One of these other mechanisms that will alter vascular stiffness but that is not a normal physiological variable is vascular geometry. The radius: wall-thickness ratio changes as a result of vasoconstriction but is not an initial cause of vasoconstriction. If, however, primarily the radius: wall-thickness ratio decreases; then the vessel will become stiffer even though the smooth muscle or other wall structures are unchanged (5).

Several investigators have shown that the water content of rat, human, and dog arteries is elevated in hypertension and have suggested that this increased water content may be associated with an increased wall thickness $(1,5)$. In this study, attempts were made to estimate the wall thickness in order to compute the $r / \delta$ and also to correlate the vessel's water content. As shown in Table I, the water content per kilogram wet weight increased $3 \%$. If the water content is computed as the percentage of change per dry solids, this represents a $12 \%$ increase with the development of hypertension. Estimates of $r / \delta$ obtained in this study and shown in Table I do not indicate that significant changes occurred. As noted above, the method is too imprecise to correlate with the water changes observed.

In evaluating the cause of the increased stiffness, two possibilities may be considered, singly, or together: 1) that changes occur within the vessel wall causing a new elastic modulus to develop, i.e., structural changes resulting in different moduli over the same range of strain; or 2) that the modulus changes because the strain changes, that is, the original wall properties were such that the elastic modulus is a function of strain; 
thus, as the pressure increases, the vessels become stretched and, as a result of the added stretch, become stiffer; in this case, the same moduli would be observed at comparable strains.

These two possibilities can be differentiated. If the normotensive moduli, over the range of pressures including the hypertensive level, are different from the hypertensive moduli over the ranges including the normotensive levels, then the differences in moduli are due to changes in the wall properties in the two states. Furthermore, if the moduli remain constant over the comparable ranges, then the pressure-diameter relationships remain linear. Conversely, if the pressure-diameter relationships remain linear over the ranges of pressure noted above, then the elastic moduli are essentially constant. Linear pressure-diameter relationships were found in 11 of 12 cases examined in this study. It should be noted in Figures 2 and 3 that the lines overlap, since the control systolic pressure was higher than the hypertensive diastolic pressure. Thus, in the region of overlap, there are two different pressurediameter plots, with different slopes for the same pressure range. Even in the one case (Figure 2) where the sensitive linearity test showed a probable nonlinearity over the entire pulse $(p<0.05)$, it would be very difficult to fit a curve to the hypertensive points that would have the same slope as the control for the overlapping pressure range. From these data, therefore, it is unlikely that the increased elastic modulus measured during hypertension is due to the vessels being stretched out to stiffer ranges by the increased pressure, especially not in the zone where control and hypertensive pressures overlap.

This is strong evidence, then, against the possibility that the changes observed before and during hypertension were due to nonlinearity. These tests of linearity confirm previous studies $(3,6)$ indicating that the elastic moduli of arteries remain essentially constant over physiological pressure ranges unless the wall properties change because of, for example, vasodilation or vasoconstriction. In other words, pressure changes of $30 \%$ occurring during each cardiac cycle are associated with small strain, i.e., a 30\% change in pressure results in a strain of approximately $2 \%$ in the femoral artery. When changes in vessel-wall properties due to reflex, humoral, or drug effects cause changes in vessel-wall properties, the changes are relatively long compared with the duration of the cardiac cycle. In this study, the relative constancy of the elastic moduli has also been observed in experimental renal hypertension.

The chemical environment of the vessel wall may also affect its mechanical properties in several ways. Changes in electrolyte concentrations may, for example, alter smooth muscle contractibility or the water content, as well as properties of connective tissue. Many investigators have observed variable changes in the electrolyte content of arteries in hypertension; particularly, the $\mathrm{Na}$, $\mathrm{K}$, and $\mathrm{Cl}$ ions have been measured. Most workers have observed an increased sodium concentration in hypertension. As shown in Tables I and II, the sodium content per unit of wet weight of the femoral artery in this study did not change significantly with the onset of hypertension.

It has been found, in the sham procedures similar to that required to measure the vessel-wall strain, that the scatter of values of the normal sodium content of the vessel wall was considerably increased when mechanical properties were measured (7). In a separate study, the chemical composition at several sites along the aorta of these same hypertensive animals was compared to a comparable normal group. Mechanical properties were not measured on the aorta, so the chemical evaluation was free from this obscuring influence. The water and sodium contents of the aortas of these hypertensive dogs were significantly elevated over normal (8).

\section{SUMMARY}

Attention has been focused on the end organ of almost all forms of hypertension, the artery. An attempt has been made to quantitate some of the changes that the artery undergoes with hypertension. The chemical and mechanical properties of femoral arteries in hypertensive dogs were studied twice: once before hypertension, and again during experimental hypertension produced by wrapping the kidneys with cellophane.

The elastic stiffness of the femoral artery was measured in vivo, and this pressure-radius relationship showed a significant increase with the development of hypertension. Evidence is presented to show that the increase in elastic stiffness 
was not due to the arterial wall's being stretched to a stiffer range because of elastic nonlinearity. The change in stiffness is probably a measurement of the change in the arterial-wall material during hypertension.

The chloride, potassium, sodium, and water contents of the same vessels were determined before and during hypertension. The water content was found to increase with increasing elastic stiffness in hypertension. The interrelationships between the elastic modulus and the chemical composition of the arterial wall are briefly considered, but no simple relation seems apparent.

\section{REFERENCES}

1. Tobian, L. Interrelationship of electrolytes, juxtaglomerular cells and hypertension. Physiol. Rev. 1960, 40, 280.
2. Page, I. H. The production of persistent arterial hypertension by cellophane perinephritis. J. Amer. med. Ass. 1939, 113, 2046.

3. Peterson, L. H., R. E. Jensen, and J. Parnell. Mechanical properties of arteries in vivo. Circulat. Res. 1960, 8, 622.

4. Bartlett, M. S. Fitting a straight line when both variables are subject to error. Biometrics 1949, 5, 207.

5. Peterson, L. H. Systems behavior, feed-back loops and high blood pressure research. Circulat. Res. 1963, 12, 585.

6. Patel, D. J., A. J. Mallos, and D. L. Fry. Aortic mechanics in the living dog. J. appl. Physiol. 1961, 16, 293.

7. Feigl, E. O., L. H. Peterson, and A. W. Jones. Unpublished observations.

8. Jones, A. W., E. O. Feigl, and L. H. Peterson. Water and electrolyte content of normal and hypertensive arteries in dogs. In press. 